\title{
Idealización: concepción estructuralista y generalización modelo-teórica*
} Idealization: Structuralist View and Model-Theoretical Generalization

Xavier de Donato Rodríguez ${ }^{\dagger}$

Marek Polanski

\begin{abstract}
Resumen
La presente contribución es un resumen de algunas ideas acerca de la idealización como relación interteórica. En primer lugar, se discuten las ideas de Leszek Nowak acerca de la idealización, la aproximación y la confirmación, las cuales han inspirado la llamada escuela de Poznań, y se ilustran por medio del ejemplo de la ley de caída libre de Galileo. Tras algunas críticas a la misma y después de una discusión acerca de distintas concepciones de la idealización basadas en la noción de deformación contrafáctica, presentamos una reconstrucción estructuralista de la noción de idealización, para luego abordar su generalización modelo-teórica. La ilustramos asimismo con ayuda del mismo ejemplo histórico. Finalmente, proponemos una caracterización de la idealización interteórica en términos de la noción tarskiana de interpretabilidad relativa.
\end{abstract}

Palabras clave: idealización - metateoría estructuralista - escuela de Poznan - aproximación - interpretabilidad relativa

\begin{abstract}
The present article is a survey of some ideas on idealization understood as an intertheoretical relation. First, Leszek Nowak's views on idealization, approximation and confirmation, that have inspired the so-called Poznań School, are discussed and illustrated with the example of Galileo's Law of Free Fall. After some criticism and discussion about different concepts of idealization in terms of counterfactual deformation, we present a structuralist reconstruction of the notion of idealization and then we turn to a model-theoretic generalization. We illustrate this by using the same historical example. Finally, we offer a characterization of intertheoretical idealization in terms of Tarski's notion of relative interpretability.
\end{abstract}

Keywords: idealization - structuralist metatheory - Poznań School - approximation - relative interpretability

\footnotetext{
* Recibido: 7 de octubre de 2012. Aceptado con revisiones: 28 de enero de 2013.

† Departamento de Lóxica e Filosofía Moral, Área de Lóxica e Filosofía da Ciencia, Universidade de Santiago de Compostela, España. Para contactar al autor, por favor, escribir a: Xavier_donato@yahoo.com.

Metatheoria 5(2)(2015): 45-55. ISSN 1853-2322. eISSN 1853-2330.

(C) Editorial de la Universidad Nacional de Tres de Febrero. Publicado en la República Argentina.
} 


\section{Introducción}

La idealización vuelve a ser un tema muy discutido en la literatura y los congresos de filosofía de la ciencia de hoy. Desde los trabajos de Nowak (1980), Nowak y Nowakowa (2000), Laymon (1982), Laymon (1987) o Cartwright (1989), la idealización ha sido un tema muy estudiado tanto desde un punto de vista formal como informal. Se trata de un tema vasto y complejo, pues el término se puede entender, y se ha entendido de hecho, de diversas maneras. Una buena muestra de las distintas aproximaciones al tema lo ofrece la serie que bajo el título "Idealization" publican los Poznań Studies in the Philosophy of Science and the Humanities con la editorial Rodopi hasta hace unos años y con la editorial Brill en el presente (catorce volúmenes hasta la fecha). Algunos de los sentidos asociados al término "idealización" -muchos de ellos relacionados entre sí- son: construcción de modelos, abstracción de parámetros o selección de factores relevantes, pensamiento contrafáctico, idealizaciones y aproximaciones involucradas en relaciones entre leyes o teorías, leyes ceteris paribus, experimentos mentales. En el presente artículo, nosotros nos vamos a detener en las idealizaciones involucradas en relaciones interteóricas (o, si se quiere, también entre leyes de una misma teoría). Este es un tema que es fuente de controversias, pues distintos casos históricos parecen poder reconstruirse desde perspectivas diferentes e incluso opuestas, con ciertas desventajas o inconvenientes en cada caso; así, por ejemplo, el caso Galileo-Newton es entendido por Nowak como un caso de idealización, por los estructuralistas como un caso de especialización y por Rott (1991) como uno de aproximación, mientras que el caso Kepler-Newton es entendido en la metateoría estructuralista como un caso de aproximación y por Rott (1991) como un caso de idealización. El estudio de la lógica de la idealización es ciertamente un capítulo importante de la filosofía de la ciencia y su aplicación requiere, obviamente, de la confrontación del análisis con los casos históricos, la cual será una prueba de fuego para cada uno de los análisis. El metateórico, estructuralista o no, debe operar bajo este presupuesto. Algunos contrargumentos y objeciones pueden, en cambio, ser a priori. En primer lugar, presentaremos de forma concisa y simplificada la esencia de la concepción nowakiana de la idealización junto con su discusión crítica. Veremos algunos puntos críticos que nos llevan a rechazarla, a pesar de estar de acuerdo con la idea básica. En el apartado siguiente, daremos una caracterización en términos estructuralistas que intenta mejorar las hasta ahora dadas. Finalmente, veremos qué motivos hay para desear una generalización modelo-teórica de la noción de idealización. Tanto la parte crítica como la parte constructiva de este trabajo está ampliamente basado en de Donato (2005). En de Donato (2011) ya presentamos un resumen y análisis de la concepción de la idealización debida a la escuela de Poznań, al tiempo que presentábamos un extenso comentario de las reconstrucciones de la misma en términos estructuralistas. El resultado principal allí defendido fue la definición de idealización en términos estructuralistas, en un modo que se halla en sintonía con la idea principal de Nowak y sus seguidores. En el presente artículo, además de presentar la concepción nowakiana con algo más de detalle y con un ejemplo, el caso Kepler-Newton, que pretende ilustrarla, queremos exponer las líneas generales de un nuevo análisis de idealización para el caso interteórico, usando asimismo el ejemplo citado. En la próxima sección, presentamos con detalle la concepción de la escuela de Poznań y la ilustramos con el mencionado ejemplo. En la sección 3, realizamos un resumen de las reconstrucciones estructuralistas, incluyendo la nuestra, presentada en de Donato (2011). Finalmente, en la sección 4 presentamos y defendemos un nuevo análisis de la idealización como relación interteórica, ampliamente basado en de Donato (2005).

\section{La concepción nowakiana de la idealización}

Nowak y sus seguidores presuponen la concepción enunciativa o clásica de las teorías científicas. Para la escuela de Poznań, las idealizaciones son básicamente enunciados de cierto tipo, con una forma 
lógica típica cuya estructura tratan de analizar en términos de lógica de primer orden. De acuerdo con esta concepción, una ley es ante todo un enunciado universalmente cuantificado (con una serie de características) y las teorías son clases de enunciados de este tipo. La concepción nowakiana del proceso de idealización-concretización puede presentarse de la siguiente forma. Partimos de una ley máximamente idealizada $\left(T_{k}\right)$ para llegar a una máximamente concretizada $\left(T_{0}\right)$. La noción de concretización, que a continuación explicaremos, es la conversa de la de idealización. Sea $x$ un "sistema real" (para utilizar las propias palabras de Nowak), donde "sistema real" significa básicamente individuos pertenecientes al universo de la teoría: ejemplos de sistemas reales son (casos particulares de) conductores en el caso de la teoría de la electricidad o gases en el caso de la teoría de los gases -es decir, representan la ontología de la teoría. Sea $F(x)$ una magnitud o cualidad de $x, H_{1}(x), \ldots, H_{n}(x)$ algunas funciones-parámetro de las que $F(x)$ necesariamente depende, $p_{k}, \ldots, p_{1}$ las funciones-parámetro que son tenidas en cuenta en las sucesivas concretizaciones de la ley más idealizada $T_{k}, f_{k}, \ldots, f_{0}$ las leyes que determinan el valor de $F(x)$, y asúmase que $x$ pertenece a la clase $R$ que constituye el dominio empírico de ese sistema y que todas las funciones tiene números reales o $n$-tuplas de números reales como valores. Cuando Nowak dice que $H(x)$ cumple el papel de un factor "necesario", está asumiendo que hay factores que no pueden ser ignorados, que necesariamente (en algún sentido de necesidad) determinan el valor de $F(x)$. Nowak no especifica qué tiene aquí en mente y, de hecho, éste es uno de los puntos críticos observados por sus críticos. El esencialismo parece ser un aspecto característico de la posición de Nowak, quien adscribe esta distinción entre factores esenciales y adventicios a Marx, considerándola una característica fundamental del método de idealización (Nowak 1980, p. 95). Este es ya un primer punto de controversia, porque parece claro que podemos defender el método de la idealización sin aceptar la mencionada tesis metafísica (o cualquier otra). De acuerdo con Nowak, hay usualmente más de un factor esencial e incluso entre los factores esenciales puede establecerse una cierta jerarquía de acuerdo a su grado de influencia (Nowak 1980, pp. 97-98). Por mor de la simplicidad, supongamos que hay uno sólo de estos factores esenciales.

Volviendo al punto de partida: la estructura de las leyes idealizadas es la de un enunciado condicional, cuyo antecedente contiene alguna idealización, que típicamente toma la forma: para cierto $x$ la función $F$ tiene el valor "límite" 0, 1 o infinito. Aquí tenemos un segundo punto de controversia, a saber, la interpretación de los condicionales. Si asumimos que son materiales, entonces tenemos el conocido problema de que son siempre (trivialmente) verdaderos (ya que las condiciones ideales nunca se cumplen). Parece, pues, más natural interpretarlos como contrafácticos (Niiniluoto 1999, p. 137). Pero esta no parece ser la interpretación de Nowak y seguidores. Por lo demás, ni él ni sus seguidores ofrecen análisis alguno de dichos contrafácticos. Aceptemos por un momento que se trata de condicionales contrafácticos. La ley más idealizada, $T_{k}$, contiene $k$ asunciones contrafácticas: $p_{k}(x)=0, \ldots$, $p_{1}(x)=0$. La siguiente, $T_{k-1}$, contendrá $k-1$ asunciones contrafácticas, etc. Introduciendo una terminología que no es de Nowak, podemos decir que $T_{i}$ es "la idealización inmediata" de $T_{i-1} \mathrm{y}$, conversamente, $T_{i-1}$ es "la concretización inmediata" of $T_{i}{ }^{1}$

(T) $F(x)=f_{k}(H(x))$.

$\left(T_{k}\right) R(x) \wedge p_{1}(x)=0 \wedge \ldots \wedge p_{k-1}(x)=0 \wedge p_{k}(x)=0 \Rightarrow F(x)=f_{k}(H(x))$.

$\left(T_{k-1}\right) R(x) \wedge p_{1}(x)=0 \wedge \ldots \wedge p_{k-1}(x)=0 \wedge p_{k}(x) \neq 0 \Rightarrow F(x)=f_{k-1}\left(H(x), p_{k}(x)\right)$.

$\left(T_{k-2}\right) R(x) \wedge p_{1}(x)=0 \wedge \ldots \wedge p_{k-2}(x)=0 \wedge p_{k-1}(x) \neq 0 \wedge p_{k}(x) \neq 0 \Rightarrow F(x)=f_{k-2}\left(H(x), p_{k}(x), p_{k-1}(x)\right)$.

$\left(T_{i}\right) R(x) \wedge p_{1}(x)=0 \wedge \ldots \wedge p_{i}(x)=0 \wedge p_{i+1}(x) \neq 0 \wedge \ldots \wedge p_{k-1}(x) \neq 0 \wedge p(x) \neq 0 \Rightarrow F(x)=f_{i}\left(H(x), p_{k}(x), \ldots, p_{i+1}(x)\right)$.

$\left(T_{0}\right) R(x) \wedge p_{1}(x) \neq 0 \wedge \ldots \wedge p_{k}(x) \neq 0 \Rightarrow F(x)=f_{0}\left(H(x), p_{k}(x), p_{k-1}(x), \ldots, p_{1}(x)\right)$

Obviamente esto no cubre todas las formas de leyes idealizadas, pero captura la idea general. Dos puntos importantes hay que notar. El primero es que, como ya hemos dicho, hemos de interpretar la

\footnotetext{
${ }^{1}$ Este no es término de Nowak, sino mío, pues interpreto la idealización como relación transitiva.
} 
conectiva ' $\Rightarrow$ ' como un condicional contrafáctico si no queremos convertir las idealizaciones en vacuamente verdaderas (ver, p.e., Niiniluoto 1999, p. 137). El segundo punto es el hecho de que lo más plausible es interpretar la relación de idealización como transitiva, es decir, $T_{k}$ es una idealización de $T_{k-1}$ pero también de $T_{k-2}$, que es la concretización inmediata de $T_{k-1}$. Parece que la caracterización de Nowak implica un orden lineal de factores que se suponen ordenados según su grado de relevancia o influencia. Pero, si mi interpretación es correcta, el análisis de Nowak es demasiado restrictivo, porque podemos negligir cualquiera de los factores considerados ignorables en un tiempo dado. La caracterización de Nowak puede asimismo ser interpretada en el sentido de una reconstrucción realizada a posteriori por el filósofo de la ciencia, que simplemente ordena los factores de acuerdo con el orden cronológico en que son descubiertos por el científico (aunque esto no es lo que dice Nowak).

De acuerdo a la concepción de la escuela de Poznań, $T_{k-1}$ converge asintóticamente en $T_{k}$ cuando para cada $x, p_{k}(x)$ tiende a cero. ${ }^{2}$ Normalmente, incluso $T_{0}$ no es satisfecha en las ciencias empíricas. El científico debe introducir correcciones destinadas a aproximar el valor de $F$. En este caso, introducimos aproximaciones de idealizaciones, en lugar de concretizaciones. En general, para cualquier idealización $T_{i}$, podemos introducir -según Nowak- una aproximación, $A T_{i}$, de la forma:

$\left(A T_{i}\right) G(x) \wedge p_{1}(x) \neq 0 \wedge \ldots \wedge p_{i}(x) \neq 0 \wedge p_{i+1}(x) \neq 0 \wedge \ldots \wedge p_{k-1}(x) \neq 0 \wedge p_{k}(x) \neq 0 \Rightarrow$

$F(x) \approx_{\varepsilon} f_{i}\left(H(x), p_{k}(x), \ldots, p_{i+1}(x)\right)$,

donde " $F(x) \approx_{\varepsilon} f_{i}\left(H(x), p_{k}(x), \ldots, p_{i+1}(x)\right)$ " significa:

" $\left|F(x)-f_{i}\left(H(x), p_{k}(x), \ldots, p_{i+1}(x)\right)\right| \leq \varepsilon "$ y $\varepsilon$ es un cierto grado de exactitud introducido por la comunidad cientifica (Nowak 1992, p. 12, 1994, p. 34).

Pero $\left(A T_{i}\right)$ no es satisfecha en general. El grado de aproximación $\varepsilon$ depende de ciertos valores límites de las funciones parámetro $p_{1}, \ldots, p_{i}$, a saber, cuando para cada $x$, se satisface " $p_{1}(x) \leq \alpha_{1} \wedge \ldots \wedge$ $p_{i}(x) \leq \alpha_{i}$ ", donde $\alpha_{1}, \ldots, \alpha_{i} \neq 0$, entonces " $F(x) \approx_{\varepsilon} f_{i}\left(H(x), p_{k}(x), \ldots, p_{i+1}(x)\right)$ ":

$$
\begin{aligned}
\left(\mathrm{AT}_{i}^{\prime}\right) \mathrm{G}(x) & \wedge p_{1}(x) \leq \alpha_{1} \wedge \ldots \wedge p_{i}(x) \leq \alpha_{i} \wedge p_{i+1}(x) \neq 0 \wedge \ldots \wedge p_{k-1}(x) \neq 0 \wedge p_{k}(x) \neq 0 \rightarrow \\
F(x) & \approx_{\varepsilon} f_{i}\left(H(x), p_{k}(x), \ldots, p_{i+1}(x)\right)
\end{aligned}
$$

donde $\alpha_{1}, \ldots, \alpha_{i} \neq 0$ y $\varepsilon \neq 0$ son ciertos grados de exactitud determinados por la comunidad científica. ${ }^{3}$ En general, cuanto más pequeños sean los valores $\alpha_{1}, \ldots, \alpha_{i}$, más pequeño es el de $\varepsilon$. Logramos tener una instancia confirmadora de $A T_{i}$, cuando para un objeto empírico dado: $\left|F(a)-f_{i}\left(H(a), p_{n}(a), \ldots, p_{i+1}(a)\right)\right|$ $\leq \varepsilon$. Diremos que $A T_{i}$ está confirmado (al menos por el momento) si tiene sólo instancias confirmadoras, esto es, si no hay un "objeto empírico" (en expresión del propio Nowak) tal que:

$$
\left|F(a)-f_{i}\left(H(a), p_{n}(a), \ldots, p_{i+1}(a)\right)\right|>\varepsilon .^{4}
$$

Para testar una ley idealizada, los científicos deben proceder de la siguiente manera:

\section{Concretizaciones}

$A T_{k}$ es confirmada $\Downarrow$

$T_{k}$ es aceptada como buena idealización

$$
\begin{gathered}
\text { 1) } A T_{k} \text { no es confirmada } \\
\Downarrow \\
T_{k-1} \text { es propuesta y su } \\
\text { aproximación } \\
A T_{k-1} \text {, debe ser testada } \\
(k+1) A T_{0} \text { no es confirmada } \\
\Downarrow
\end{gathered}
$$

No hay más concretizaciones disponibles

y $T_{k}$ debe ser rechazada.

\footnotetext{
${ }^{2}$ Nowak no especifica ninguna métrica o topología.

${ }^{3}$ Nowak (1980, cap. 10, pp. 159-160). Ver Nowakowa (1994, p. 56), y Kupracz (1992, p. 129).

${ }^{4}$ Para más detalles sobre la confirmación de idealizaciones desde esta perspectiva, ver Patryas (1977), Paprzycka (1992), Kupracz (1992), Nowak (1992, p. 15, 1994, cap. IV).
} 
Nótese que, como apunta Niiniluoto (1989), si el principio de correspondencia es satisfecho, es decir, si para $j=1, \ldots, k$, se cumple lo siguiente:

$$
\lim _{z \rightarrow 0} f_{j}\left(H(x), p_{1}(x), \ldots, p_{j-1}(x), z\right)=f_{j-1}\left(H(x), p_{1}(x), \ldots, p_{j-1}(x)\right)
$$

entonces cada ley $T_{i}$ implica las leyes más idealizadas, de manera que $T_{j}$ implica $T_{k}, \ldots, T_{j+1}$ (para cada $j=$ $\left.0, \ldots, k_{-1}\right)$. Todos los modelos de $T_{j}$ también son modelos de $T_{j+1}:\left\|T_{0}\right\| \subseteq\left\|T_{1}\right\| \subseteq \ldots \subseteq\left\|T_{k}\right\| .^{5}$

Daremos ahora un ejemplo de ley idealizada, la ley de caída libre de Galileo [la ley de caída libre, su concreción dentro de la mecánica galileana (cuando la velocidad inicial difiere de cero) y su "derivación" como idealización de la mecánica newtoniana]. Hemos introducido algunas modificaciones en las reconstrucciones de Nowak. De acuerdo con su notación, la ley de Galileo se puede reconstruir de la siguiente manera:

$$
f b(x, e) \wedge r(x)=0 \wedge v_{0}=0 \wedge g=\text { const } \rightarrow s(x)=1 / 2 g t(x)^{2},
$$

donde $f b(x, e)$ significa " $x$ es un cuerpo en caída libre sobre la superficie de la Tierra (e)", vo es la velocidad inicial, $r$ es la resistencia del aire, $s$ es la distancia cubierta por el cuerpo, $g$ es la constante de aceleración gravitacional y $t$ es el tiempo de caída.

La concretización de esta ley en términos de la velocidad inicial, esto es, asumiendo que la velocidad inicial es distinta de cero, es como sigue:

$$
f b(x, e) \wedge v_{0}(x)>0 \wedge r(x)=0 \wedge g=\text { const } \rightarrow s(x)=v_{0}(x) t(x)+1 / 2 g(x) t(x)^{2} .
$$

Ahora podemos ver cómo derivar la ley de caída libre como idealización de la mecánica newtoniana. Asumamos que la Tierra es una esfera perfecta y homogénea de radio $R$ y masa $M$. La ley de la gravitación de Newton implica que la Tierra atrae al cuerpo $x$ de masa $m(x)$ que se encuentra a una altura $h(x)$ de la superficie terrestre con la fuerza:

$$
-\mathrm{G} \frac{M \cdot m(x)}{(R+h(x))^{2}},
$$

donde $\mathrm{G}$ es la constante de gravitación universal. El cuerpo cae entonces con una aceleración que debe ser igual a:

$$
-\frac{\mathrm{GM}}{(R+h(x))^{2}}
$$

donde la asunción de que $h(x)=0$ da como resultado:

$$
-\frac{\mathrm{GM}}{R^{2}} \text {. }
$$

Sólo necesitamos introducir la segunda ley y resolver la siguiente ecuación:

$$
m(x) \frac{d^{2} s(x, t)}{d t(x)^{2}}=-m(x) \frac{G M}{(R+h(x))^{2}}
$$

cuya solución es:

$$
s(x)=-\frac{\mathrm{GM}}{2(R+h(x))^{2}} t(x)^{2},
$$

la cual, si $h(x) \rightarrow 0$, se reduce a la ley de Galileo, que puede verse entonces como una idealización de la ley newtoniana. Es decir, en el límite $h(x)=0$ :

$$
s(x)=-\frac{G M}{2 R^{2}} t(x)^{2}=-\frac{g t(x)^{2}}{2},
$$

\footnotetext{
${ }^{5}$ \|T $\mid$ es la clase de todos los modelos de T. Ver Niiniluoto (1989, p. 43, y 1999, p. 137).
} 
lo cual corresponde a la ley de Galileo. Explicar así las cosas no está exento de controversia, porque ¿tiene de verdad sentido que hagamos la idealización de que la altura de caída sea cero? Algunos autores creen, en consecuencia, que el caso de la ley galileana y la mecánica newtoniana es más un ejemplo de aproximación que de idealización (ver, p.e., Rott 1991, p. 292, n. 39). En Nowak (2000, pp. 46-48), se formula otra forma concretizada de la ley de caída libre tomando en cuenta la velocidad angular de la Tierra.

En de Donato (2011) se resumen los problemas que vuelven insatisfactoria la concepción de la escuela de Poznań de la idealización. Como se argumenta en ese artículo, esta concepción se enmarca dentro de la noción clásica de las teorías científica, con lo cual hereda los problemas que los estructuralistas detectaban en ésta. Aparte de que su análisis no es suficientemente general (puesto que los parámetros ignorados pueden tomar valores distintos de 0) ni tampoco suficientemente preciso (pues no nos dice, por ejemplo, cómo están relacionadas las funciones $f_{k}$ y $f_{k-1}$ ), presupone además un orden lineal de parámetros de acuerdo a su influencia y un cierto esencialismo de factores. Otro importante inconveniente es que, dado que las condiciones ideales son manifiestamente falsas, no se puede usar el condicional material para formalizar una ley idealizada (como aparentemente hacen los seguidores de esta escuela), sino que se debe usar un condicional contrafáctico. Esto lleva al conocido problema de dar una semántica adecuada para los contrafácticos. Nowak y sus seguidores no nos ofrecen ninguna. Por otro lado, Nowak y sus seguidores no parecen reconocer el importante papel de la aproximación como relación entre leyes y entre teorías, sino que para ellos es más bien un método de comparación de los valores de los parámetros que aparecen en las leyes. Y, finalmente, el análisis de Nowak no parece capturar suficientemente bien la diferencia entre una idealización interteórica y una intrateórica. En ocasiones, su análisis parece ser más un intento de caracterizar la relación de idealización intrateórica. En de Donato (2005) y de Donato (2011) tratamos de presentar una noción estructuralista de idealización para el caso intrateórico y, muy especialmente, para el caso interteórico. ${ }^{6}$

\section{Reconstrucción estructuralista}

Comparaciones entre la escuela de Poznań y la estructuralista han sido realizados ya por Kuokkanen (1988), Hamminga (1989), Kuokkanen y Tuomivaara (1992), Ibarra y Mormann (1994) y Balzer y Zoubek (1994). En de Donato (2011, pp. 78-80) se analiza la noción de estructura idealizacional de Ibarra y Mormann definida en términos de operadores de deformación contrafáctica y se dan algunas razones por las cuales dicha noción no nos parece generalizable a casos de relación interteórica. También allí, y en de Donato (2005), presentamos nuestra propia reconstrucción estructuralista de la idealización nowakiana más un resultado formal, en parte basados en la reconstrucción de Balzer y Zoubek (1994).

Como allí decíamos, el punto esencial en nuestra reconstrucción estructuralista de la concepción de Poznań es que en lugar de clases de fórmulas tenemos clases de modelos. Los modelos de la teoría concretizada contienen la función-parámetro que es tenida en cuenta por primera vez, mientras que en los modelos de la teoría idealizada correspondiente esta misma función-parámetro se vuelve una función constante que siempre asume el valor 0 (o algún otro valor límite), resultando así prescindible. El proceso de concretización puede ser reconstruido, pues, de la siguiente manera: podemos obtener la teoría concretizada $\mathrm{T}^{\prime}$ como la clase de estructuras que toman como nueva función-parámetro exactamente aquella que ha sido ignorada en la teoría idealizada correspondiente $\mathrm{T}$, que es vista como la reducción, mediante una función llamada red, de la clase de estructuras $\mathrm{T}^{\prime}$. Esta transición debe satisfacer además ciertas condiciones especificadas. Entre ellas que las aplicaciones intencionales de la teoría idealizada son exactamente las mismas que las de la teoría concretizada, excepto sólo que esta segunda teoría toma un nuevo factor en cuenta, de modo que la teoría concretizada tiene más aplicaciones que la idealizada. Se exige también que la teoría concretizada y la idealizada son "dramáticamente" diferentes con respecto a sus modelos actuales, es decir, que los axiomas principales

${ }^{6}$ Para otras críticas a la concepción nowakiana, ver Krajewski (1977, cap. 1) y Hasse (1995, cap. 2, sec. 2.1.4, en particular p. 101). 
se contradicen, aunque coincidan con respecto a sus modelos potenciales (los modelos potenciales de la teoría idealizada son simples reducciones, mediante una función $r$, de los modelos potenciales de la teoría concretizada). Esto es así porque de acuerdo con la teoría concretizada no es posible para un sistema real ser modelo de la teoría concretizada sin tener en cuenta cierto factor relevante, que no era tenido en cuenta por la teoría idealizada. Finalmente, se requiere además que se cumpla una condición para relacionar apropiadamente los modelos actuales de ambas teorías. Para los detalles, véase de Donato (2011, pp. 82-85). Lo que a continuación vamos a exponer pretende ser un nuevo análisis de la idealización como relación interteórica que va más allá del ofrecido en de Donato (2011) y que toma ideas presentadas en de Donato (2005). La motivación se explica al comienzo de la siguiente sección.

\section{Idealización interteórica: un nuevo análisis}

La principal motivación de un nuevo análisis es triple:

(1) la necesidad de una caracterización formal que haga preciso lo que haya de correcto en las intuiciones de Nowak y otros y que no parece expresado de modo suficientemente apropiado; es asimismo importante precisar la relación entre idealización y aproximación;

(2) la idea de encontrar una conexión enttre la concepción aproximativa (defendida, entre otros, por los estructuralistas (Balzer, Moulines \& Sneed 1987, Balzer, Pearce \& Schmidt 1984) y Pearce y Rantala (1983, 1984a, 1984b), entre otros) y la que podemos llamar "idealizacional" (escuela de Poznań, Rott y Barr, entre otros);

(3) el propósito de evitar un análisis en términos de contrafácticos como el único análisis posible de las idealizaciones en la ciencia.

El análisis que presentamos en esta última sección, aunque substancialmente diferente, estuvo inicialmente inspirado en algunos hallazgos que se pueden rastrear en Barr (1971). Junto con Nowak (1972), Barr (1971) y (1974) ha sido uno de los primeros en preocuparse por la lógica de las idealizaciones. Aunque los análisis de Barr y Nowak son primariamente sintácticos (siguen la concepción enunciativa de las teorías), están interesados no sólo en un análisis meramente sintáctico, sino también en los aspectos semánticos y pragmáticos. No solo es la idealización, sino su relación con la explicación y la confirmación de teorías lo que es objeto de sus investigaciones. Por otro lado, un análisis de la idealización en términos puramente sintácticos no es posible. Un primer punto en el que los análisis de Nowak y Barr difieren es que Barr no nos ofrece, al contrario que Nowak, un análisis de la relación conversa de la idealización, la concretización, la cual cumple un papel importante en el desarrollo de la ciencia.

Una desventaja compartida por Nowak y Barr es que ninguno de los dos proporciona una caracterización precisa de la lógica de la idealización. Mientras Nowak no presenta un análisis adecuado de los condicionales involucrados en las idealizaciones y de las relaciones entre los lenguajes y teorías implicados en casos de idealización interteórica, el análisis de Barr es insuficiente y ambiguo. No obstante, Barr presenta la ventaja de ofrecernos un análisis que no requiere de contrafácticos.

El análisis de Rott $(1991,1994)$ de la idealización sí hace uso de los contrafácticos, aunque es bastante más preciso, al basarse en la teoría formal del cambio de creencia de Gärdenfors, que Rott aplica al cambio no monotónico y la revisión de teorías. Nuestro propio análisis es un intento, como avanzábamos más arriba, de recoger aquellas intuiciones de Nowak, Barr o Rott que nos parecen correctas, pero tratándolas desde una perspectiva muy diferente, a saber, por medio de la lógica de primer orden y la teoría de modelos, perspectiva que puede verse a su vez como una generalización modeloteórica de los análisis estructuralistas, pudiéndose ver así -según nuestra opinión- cuáles son las interconexiones entre las distintas concepciones de la idealización que se han mencionado. ${ }^{7}$

Recordemos las dos ecuaciones mencionadas en la sección 2 de esta contribución, una en la que la posición está calculada de acuerdo con la segunda ley y la ley de gravitación newtonianas:

\footnotetext{
${ }^{7}$ Este no es lugar, sin embargo, para un análisis comparativo de las distintas concepciones; para ello remitimos a de Donato (2005).
} 
$[\mathrm{NFF}]$

$$
s(x)=-\frac{\mathrm{GM}}{(R+h(x))^{2}}(t(x))^{2}
$$

y otra, que es la formulación newtoniana de la ley galileana cuando $h \rightarrow 0$ :

[GFF]

$$
s(x)=-\frac{G M}{2 R^{2}} t(x)^{2}=-1 / 2 g t(x)^{2}
$$

que corresponde a la formulación de Galileo en Dos nuevas ciencias (Cohen 1985, cap. 5, n. 2).

Para la derivación de la ecuación galileana la constante $\frac{G M}{R^{2}}$ ha sido sustituida por lo que llamamos hoy la constante de aceleración $g$, que en las cercanías de la superficie terrestre equivale (aproximadamente) a $9,81 \mathrm{~m} / \mathrm{s}^{2}$. Pero, como es bien sabido, el valor de la aceleración de un cuerpo en caída libre aumenta conforme la altura decrece. La ecuación newtoniana correspondiente es:

$$
m \frac{d^{2} s(x)}{d t(x)^{2}}=-m \frac{G M}{(R+h(x)-s(x))^{2}}
$$

cuya resolución da valores más pequeños que [NFF] pero mayores que [GFF].

Inspirándonos en Barr (1971), tratamos a continuación de ofrecer un análisis que recoja sus intuiciones, pero evite las dificultades e imprecisiones lógicas. La intuición básica es que debe haber dos fórmulas en el lenguaje de la nueva teoría (la de Newton en nuestro caso), una cuya clausura existencial es falsa y, en casos extremos, incompatible con la teoría (en nuestro caso, esta fórmula dice que la altura o distancia de caída es igual a 0 , lo que es perfectamente posible en la teoría, pero que no tiene sentido) y una fórmula correspondiente cuya clausura existencial no es probada por la teoría, pero sí es perfectamente compatible con ella (en nuestro caso, esta fórmula establece que el cuerpo cae en las cercanías de la superficie terrestre). Para $h=0$, la ley de caída libre se cumple (asumiendo incluso la teoría de gravitación newtoniana), pero obtenemos una forma inútil de la ley. Este fue el principal error del análisis Eberle (1971), en una de cuyas intuiciones -como veremos- también nos inspiramos ligeramente. En lugar de eso, lo que tenemos es que para $h$ suficientemente pequeña la diferencia entre los valores arrojados por las ecuaciones de Galileo y Newton son también negligibles, de modo que la ley de Galileo puede seguir siendo usada legítimamente. Necesitamos, sin embargo, una formulación precisa de estas intuiciones y creemos que la mejor manera de hacerlo es recurrir a la teoría de modelos.

Sean $L, L^{\prime}$ lenguajes de primer orden en los que las teorías $\mathrm{T}$ (la teoría idealizada, en nuestro caso la de Galileo) y $\mathbf{T}^{\prime}$ (la más concretizada, la de Newton en nuestro caso) están, respectivamente, formulados. Asumamos, por el momento, que $L \subseteq L^{\prime}$ (en nuestro caso podemos asumir que $\frac{G M}{R^{2}}$, por lo que $T^{\prime}$ no será exactamente la teoría newtoniana, sino una extensión definicional de la misma, en la que $g$ es introducida como abreviatura de $\frac{G M}{R^{2}}$. Podemos asumir que $\mathbf{T}$ y $\mathbf{T}^{\prime}$ son reconstrucciones estructuralistas de las teorías, i.e. $\mathbf{T}=\left\langle\mathbf{M}_{\mathrm{p}}, \mathbf{M}, \ldots\right\rangle, \mathrm{y} \mathrm{T}^{\prime}=\left\langle\mathbf{M}_{\mathrm{p}}^{\prime}, \mathbf{M}^{\prime}, \ldots\right\rangle$, que $L, L^{\prime}$ son lenguajes de primer orden para $\mathbf{T}$ y $\mathrm{T}^{\prime}$ respectivamente y que $\Sigma \subseteq \operatorname{Sent}(L)$ y $\Sigma^{\prime} \subseteq \operatorname{Sent}\left(L^{\prime}\right)$ son las clases de axiomas para $\mathbf{T}$ y $\mathbf{T}^{\prime}$ respectivamente, de modo que $\mathbf{M}=\operatorname{Mod}(\Sigma)$ y $\mathbf{M}^{\prime}=\operatorname{Mod}\left(\Sigma^{\prime}\right)$. Es importante considerar solo aquellas estructuras que incluyen el conjunto de los números reales y los elementos del análisis matemático, con todo lo necesario para formular y resolver diferenciales e integrales. En tales estructuras debe estar fijada la interpretación estándar del lenguaje del análisis. También podríamos considerar la clase de aplicaciones intencionales, pero, por mor de la simplicidad, la hemos omitido. $" \operatorname{Mod}(\Sigma) \vDash \sigma$ " significa que $\sigma$ es verdadero en todos los modelos de $\Sigma$. Cuando éste no es el caso, diremos que $\sigma$ es falsa en $\operatorname{Mod}(\Sigma)$, i.e. $\sigma$ no es verdadero en todos los modelos de $\Sigma$, y escribiremos " $\operatorname{Mod}(\Sigma) \vDash /=\sigma$ ". Algo más fuerte es afirmado si escribimos " $\operatorname{Mod}(\Sigma) \vDash \neg \sigma$ ", a saber que en todos los modelos de $\Sigma$ se sigue la negación de $\sigma$. Cuando éste es el caso, se dice que $\sigma$ contradice los axiomas de 
$\mathrm{T}^{\prime}$ (algunos de ellos). Sea $\Gamma^{\prime} \subset \Sigma^{\prime}$ la subclase de axiomas que son contradichos por la idealización en cuestión y sea $\operatorname{Mod}\left(\Gamma^{\prime}\right)$ la clase de sus modelos. En el caso Galileo-Newton, es claro que la suposición $h$ $=0$ no contradice ninguno de los axiomas de Newton, aunque no tiene, por así decirlo, sentido. Lo que hacemos es simplemente restringir el dominio de aplicación de nuestra teoría. Una ley (como la de Galileo) puede seguir siendo usada como una buena aproximación dentro de cierto dominio (distancias en la cercanía de la superficie terrestre). En el caso de algunas de las idealizaciones involucradas en el caso Kepler-Newton o en la relación entre la teoría del calor y los principios de la mecánica estadística, las idealizaciones sí contradicen algunos principios de las nuevas y más precisas teorías. En otros casos, la suposición es meramente contraria a los "hechos aceptados" o al conocimiento presupuesto por la teoría, si bien no contradice ninguno de los principios o axiomas de ésta. En un análisis apropiado de la lógica de la idealización en casos interteóricos, todos estos casos deben ser contemplados y distinguidos entre sí. La versión que ofrecemos es, pues, una bastante simplificada. Contemplaremos, para esta versión, el caso en que la "condición ideal" es compatible con la teoría, como ocurre en el caso Galileo-Newton. Para simplificar la caracterización formal, este tipo de idealización interteórica se puede formalizar como una relación intrateórica entre leyes, una idealizada y la otra concretizada, de una misma teoría:

\section{Definición 1:}

Sea $\Sigma$ una teoría formulada en el lenguaje $L$. Sean $F(\bar{x}), G(\bar{x})$ y $G^{*}(\bar{x})$ términos complejos en $L$. La fórmula “ $\forall \bar{x}(F(\bar{x})=G(\bar{x}))$ " idealiza la fórmula “ $\forall \bar{x}\left(F(\bar{x})=G^{*}(\bar{x})\right)$ " en T si y sólo si las siguientes condiciones son satisfechas:

(1) $\operatorname{Mod}(\Sigma) \vDash \neg \forall \bar{x}(F(\bar{x})=G(\bar{x}))$

(2) $\operatorname{Mod}(\Sigma) \vDash \forall \bar{x}\left(F(\bar{x})=G^{*}(\bar{x})\right)$

(3) Hay una $L(\mathrm{~T})$-fórmula de la forma " $f(\bar{x})=k$ " tal que:

(i) $\operatorname{Mod}(\Sigma+\exists \bar{x}(f(\bar{x})=k)) \neq \varnothing$

(ii) $\operatorname{Mod}(\Sigma) \vDash \forall \bar{x}(f(\bar{x})=k \rightarrow F(\bar{x})=G(\bar{x})))$

(iii) $\operatorname{Mod}(\Sigma) \vDash \forall a \exists b \forall \bar{x}\left(|f(\bar{x})-k|<b \rightarrow\left|G(\bar{x})-G^{*}(\bar{x})\right|<a\right.$

La condición (1) expresa el hecho de que la ley idealizada no es válida en $\mathrm{T}$, en nuestro caso que la ley galileana es falsa en la teoría de Newton. (2) enuncia el hecho de que la ley "concretizada" es válida en $\mathrm{T}$, es decir, en nuestro caso que la correspondiente ecuación newtoniana es válida en la teoría de Newton. (3) expresa el hecho de que hay, sin embargo, una condición ideal bajo la cual la ley galileana es aproximadamente verdadera en la teoría de Newton. La condición (i) de (3) expresa, en nuestro caso, que la condición límite " $h=0$ " es compatible con la teoría newtoniana, si bien $\mathrm{T} \vDash /=\exists \bar{x}(f(\bar{x})=$ k). La condición (ii) expresa que bajo esa condición límite la ley idealizada sería válida y (iii) expresa que hay una condición aproximada correspondiente, a saber, en nuestro caso que la altura de caída es en las cercanías de la superficie terrestre, tal que la ley idealizada (la ley de Galileo) arroja valores muy próximos a los de la ley concretizada (la ecuación newtoniana).

En los casos en que la condición límite o ideal es incompatible con T, la condición (i) de (3) no se cumple, es decir, la conjunción de los modelos de T y los modelos de la condición ideal sí es vacía. En tales casos, contemplaremos la restricción de los modelos de $\mathbf{T}$ respecto de aquellos axiomas $(\Gamma)$ que son contradichos por la condición ideal $\varphi$, de forma tal que la conjunción de esa restricción y los modelos de la condición ideal no sea vacía y la ley idealizada $\psi$ pueda valer en esa conjunción:

$$
\operatorname{Mod}(\Sigma-\Gamma) \cap\|\{\varphi\}\| \vDash \forall \bar{x} \psi(\bar{x})
$$

Esta caracterización permite un estudio comparativo entre las distintas concepciones aproximativas, incluyendo la estructuralista, y las concepciones idealizacionales. ${ }^{8}$

\footnotetext{
${ }^{8}$ Ver para los detalles de Donato (2005).
} 
Para los casos en que tenemos dos teorías cuyos lenguajes son disjuntos, es decir: $L(T) \cap L\left(T^{\prime}\right)=$ $\varnothing$, una caracterización alternativa se inspira en la noción de traducibilidad entre lenguajes, básicamente lo que corresponde al concepto tarskiano de interpretabilidad relativa, ya sugerido por Eberle (1971) para tratar casos de idealización interteórica y, más recientemente, por Niebergall (2000) y por Polanski (2002) para caracterizar la noción de reducción entre teorías. Si el concepto de interpretación relativa es adecuado como paráfrasis de traducibilidad en el caso de las idealizaciones interteóricas es algo que planteamos aquí como hipótesis a tratar con más detalle en otras publicaciones. Así, sugerimos definir:

\section{Definición 2:}

T es una idealización de $\mathbf{T}^{\prime}$ si y sólo si hay una traducción (una interpretación relativa) $f$ de $L(T)$ en $L\left(\mathrm{~T}^{\prime}\right)$ tal que para cada axioma $\varphi$ de $\mathrm{T}$ una de las siguientes dos condiciones se cumple:

(i) hay un teorema $\psi$ de $\mathrm{T}^{\prime}$ tal que $f(\varphi)$ idealiza (en el sentido de la Def. 1) $\psi$ en $\mathrm{T}^{\prime}, \mathrm{y}$

(ii) $f(\varphi)$ es un teorema de $\mathbf{T}^{\prime}$.

La comprobación de la adecuación de esta propuesta de definición cae fuera de los límites de este trabajo. Remitimos al lector a de Donato (2005), donde estas cuestiones están tratadas con algo más de detalle. Sólo queremos terminar con una observación importante, a saber, que las definiciones 1 y 2 deben ser tomadas más como modelos explicativos o caracterizaciones formales de la noción de idealización en el sentido que aquí se ha visto que como condiciones necesarias y suficientes para las idealizaciones interteóricas.

\section{Bibliografía}

Balzer, W., Pearce, D. y H.J. Schmidt (eds.) (1984), Reduction in Science. Structure, Examples, Philosophical Problems, Dordrecht: Reidel.

Balzer, W. y G. Zoubek (1994), “Structuralist Aspects of Idealization”, en Kuokkanen (1994), pp. 57-79.

Barr, E. W. (1971), “A Syntactic and Semantic Analysis of Idealization in Science”, Philosophy of Science 38: 258-272.

Barr, E. W. (1974), "A Pragmatic Analysis of Idealization in Physics”, Philosophy of Science 41: 48-64.

Brzeziński, J., Coniglione, F., Kuipers, T. y L. Nowak (eds.) (1990a), Idealization I: General Problems (Poznań Studies in the Philosophy of the Sciences and the Humanities, vol. 16), Amsterdam/Atlanta, GA: Rodopi.

Brzeziński, J., Coniglione, F., Kuipers, T. y L. Nowak (eds.) (1990b), Idealization II: Forms and Applications (Poznań Studies in the Philosophy of the Sciences and the Humanities, vol. 17), Amsterdam/Atlanta, GA: Rodopi.

Brzeziński, J. y L. Nowak (eds.) (1992), Idealization III: Approximation and Truth (Poznań Studies in the Philosophy of the Sciences and the Humanities, vol. 25), Amsterdam/Atlanta, GA: Rodopi.

Cartwright, N. (1989), Nature's Capacities and their Measurement, Oxford: Clarendon Press.

Cohen, I. B. (1985), The Birth of a New Physics, New York: W. W. Norton \& Co.

De Donato Rodríguez, X. (2005), Idealization and the Growth of Physics, Tesis doctoral, Munich: University of Munich.

De Donato Rodríguez, X. (2011), "Idealization within a Structuralist Perspective”, Metatheoria 1(2): 65-90.

Eberle, R. A. (1971), "Replacing one Theory by Another under Preservation of a Given Feature", Philosophy of Science 38: 486-501.

Haase, M. (1995), Galileische Idealisierung. Ein pragmatisches Konzept, Berlin: Walter de Gruyter.

Hamminga, B. (1989), "Sneed versus Nowak: An Illustration in Economics”, Erkenntnis 30: 247-265.

Ibarra, A. y T. Mormann (1994), "Counterfactual Deformation and Idealization in a Structuralist Framework", en Kuokkanen (1994), pp. 81-94. 
Krajewski, W. (1977), Correspondence Principle and Growth of Science, Dordrecht: Reidel.

Kuokkanen, M. (ed.) (1994), Idealization VII: Structuralism, Idealization and Approximation (Poznań Studies in the Philosophy of the Sciences and the Humanities, vol. 42), Amsterdam/Atlanta, GA: Rodopi.

Kuokkanen, M. y T. Tuomivaara (1992), "On the Structure of Idealizations. Explorations in the Poznań School Methodology of Science”, en Brzeziński \& Nowak (1992), pp. 67-102.

Kupracz, A. (1992), "Testing and Correspondence”, en Brzeziński y Nowak (1992), pp. 127-144.

Laymon, R. (1982), "Scientific Realism and the Hierarchical Counterfactual Path from Data to Theory", en Asquith, P. y T. Nickles (eds.), Proceedings of the 1982 Biennal Meeting of the Philosophy of Science Association, Michigan: East Lansing, vol.1, pp. 107-121.

Laymon, R. (1987), "Using Scott Domains to Explicate the Notions of Approximate and Idealized Data", Philosophy of Science 54: 194-221.

Niebergall, K.-G. (2000), “On the Logic of Reducibility: Axioms and Examples”, Erkenntnis 53: $27-61$.

Niiniluoto, I. (1990), “Theories, Approximations, and Idealizations”, en Brzeziński et al. (1990a), pp. 9-57.

Niiniluoto, I. (1999), Critical Scientific Realism, Oxford: Oxford University Press.

Nowak, L. (1972): "Laws of Science, Theories, Measurement (Comments on Ernest Nagel's The Structure of Science)", Philosophy of Science 39: 533-547.

Nowak, L. (1990), "Abstracts Are Not Our Constructs. The Mental Constructs Are Abstracts”, en Brzeziński et al. (1990b), pp. 193-206.

Nowak, L. (1980), The Structure of Idealization, Dordrecht: Reidel.

Nowak, L. (1992), "The Idealizational Approach to Science: A Survey”, en Brzeziński et al. (1992), pp. 9-63.

Nowak, L. y I. Nowakowa (eds.)(2000), Idealization X: The Richness of Idealization (Poznań Studies in the Philosophy of the Sciences and the Humanities, vol. 69), Amsterdam/Atlanta, GA: Rodopi.

Nowakowa, I. (1994), Idealization V: The Dynamics of idealization (Poznan Studies in the Philosophy of the Sciences and the Humanities, vol. 34), Amsterdam/Atlanta, GA: Rodopi.

Paprzycka, K. (1992), "Why do Idealizational Statements Apply to Reality?", en Brzeziński \& Nowak (1992), pp. 145. 156.

Patryas, W. (1977), The Sense of Empirical Testing (Poznań Studies in the Philosophy of the Sciences and the Humanities, vol. 3), Amsterdam: Grüner, pp. 180-198.

Pearce, D. y V. Rantala (1983), "Correspondence as an Intertheory Relation”, Studia Logica 42: 363-371.

Pearce, D. y V. Rantala (1984a), “A Logical Study of the Correspondence Relation”, Journal of Philosophical Logic 13: 47-84.

Pearce, D. y V. Rantala (1984b), "Limiting Case Correspondence between Physical Theories", en Balzer, Pearce \& Schmidt (1984), pp. 153-185.

Polanski, M. (2002), Zur logischen Analyse von Theorienreduktion und Theorienäquivalenz, Munich: Centrum für Informations- und Sprachverarbeitung.

Rott, H. (1990), “Approximation versus Idealization: the Kepler-Newton Case”, en Brzeziński et al. (1990b), pp. 101. 124.

Rott, H. (1994), Reduktion und Revision. Aspekte des nichtmonotonen Theorienwandels, Frankfurt: Peter Lang. 